\title{
Evidence for Unfolding and Refolding of Gas-Phase Cytochrome $c$ Ions in a Paul Trap
}

\author{
Ethan R. Badman,* Sunnie Myung, and David E. Clemmer \\ Department of Chemistry, Indiana University, Bloomington, Indiana, USA
}

The folding pathways of gas-phase cytochrome $c$ ions produced by electrospray ionization have been studied by an ion trapping/ion mobility technique that allows conformations to be examined over extended timescales (10 ms to $10 \mathrm{~s}$ ). The results show that the +9 charge state emerges from solution as a compact structure and then rapidly unfolds into several substantially more open structures, a transition that requires $30-60 \mathrm{~ms}$; over substantially longer timescales $(250 \mathrm{~ms}$ to $10 \mathrm{~s})$ elongated states appear to refold into an array of folded structures. The new folded states are less compact than those that are apparent during the initial unfolding. Apparently, unfolding to highly open conformations is a key step that must occur before +9 ions can sample more compact states that are stable at longer times. (J Am Soc Mass Spectrom 2005, 16, 1493-1497) (c) 2005 American Society for Mass Spectrometry

$\square$ haracterizing the nature and roles of intermediates that are formed as a protein folds is central to understanding the folding problem [1-3]. Recently, structural studies of ions in the gas phase have attracted significant attention as a means of studying intrinsic interactions of biomolecules in the absence of solvent to further understand fundamental aspects of protein folding. Examination of gas-phase structure/ conformations of protein ions has primarily been studied by ion mobility $[4,5]$ and gas-phase hydrogendeuterium exchange [6-10] methods, with some recent studies $[11,12]$ using the "gentle" dissociation process of electron capture dissociation (ECD) [13]. In addition, unfolding and folding transitions of gas-phase ions have been studied by exciting the ions via laser irradiation $[8,11,12]$ or energetic collisions $[5,8,9]$ followed by analysis of structure/conformation by the aforementioned methods. The advantages of studying gas-phase ions is that one is able to study not only "naked" ions' intrinsic physical and chemical properties, but also the stepwise solvation of these ions [14-16].

We have recently developed a new method for studying the time-dependent behavior of gas-phase protein ions. In this approach we store ions in a Paul geometry ion trap for variable amounts of time and follow the structural transitions by analyzing the conformations of electrosprayed ions by a combined ion

Published online July 12, 2005

Address reprint requests to David E. Clemmer, Department of Chemistry, Indiana University, 800 East Kirkwood Avenue, Bloomington, IN 47405. E-mail: clemmer@indiana.edu

* Current address: Department of Chemistry, Iowa State University, Ames, IA 50011, USA. mobility/time-of-flight analysis [17, 18]. In this study we show evidence for structural transitions of the +9 charge state of cytochrome $c$ that involve unfolding and refolding while ions are stored in the ion trap. The gas-phase structure of cytochrome $c$ has been the subject of a number of published reports $[5,8,9,11]$. The following data provide a look at some transitions and timescales that may be general to those found for proteins stored in ion traps. Such insight may eventually be useful in understanding fragmentation pathways-especially those involving nonergodic excitation methods.

\section{Experimental}

Equine cytochrome $c$ (>95\% purity; Sigma, St. Louis, $\mathrm{MO})$ ions were produced by electrospraying a $20-\mu \mathrm{M}$ solution (in 49\%:49\%:2\%, water/methanol/acetic acid) at atmospheric pressure. Ions enter a differentially pumped desolvation region (2.0 torr) before being extracted into a vacuum region and focused into a quadrupole ion trap (R.M. Jordan, Grass Valley, CA) containing $10^{-3}$ torr of $300 \mathrm{~K} \mathrm{He}$. Experiments are initiated by accumulating a 5-ms ion packet in the trap. These ions are stored for a variable time (ranging from $10 \mathrm{~ms}$ to $10 \mathrm{~s}$ ). After the defined storage time they are ejected into a drift tube/quadrupole mass spectrometer for analysis $[17,19]$. The drift tube contains 2.0 torr of 300 $\mathrm{K}$ He buffer gas. The pulse of ions drifts through the gas under the influence of a uniform electric field and different conformations (for a specific charge state selected by the quadrupole mass spectrometer) are separated because of differences in their collision cross 
sections with the buffer gas [20]. Compact conformers have smaller cross sections (and thus shorter drift times) than elongated conformers. In these studies, the drift times ( $\sim 3-5 \mathrm{~ms})$ are orders of magnitude shorter than the storage times; thus, the transitions that are observed are caused by changes in structure that occur in the trap. Minor changes in structure that might occur during the 3- to 5-ms drift times would have no major effect on the observed transitions because, as the data show, the transitions take 10 's of milliseconds or more to occur.

Compared with the previous study of cytochrome $c$ using this ion trapping/ion mobility method, [17], we have expanded the storage time over which the ions can be observed from $200 \mathrm{~ms}$ to $10 \mathrm{~s}$. In addition, these experiments have been performed while minimizing the injection energy from the ion trap to the drift tube to minimize structural transitions resulting from collisional heating $[4,21]$ as ions enter the drift tube. Previous studies of the time required for ions to equilibrate to a temperature in an ion trap have been performed by McLuckey and his collaborators [22].

\section{Results and Discussion}

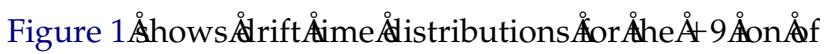
cytochrome $c$ at ion trap storage times of 20, 250, and $5000 \mathrm{~ms}$. The peaks that are observed correspond to ions having collision cross sections that agree with values reported previously for this charge state. We have labeled the peaks B $\left(\Omega=1530 \AA^{2}\right), C(\Omega=1700$ $\left.\AA^{2}\right)$, D $\left(\Omega=1960 \AA^{2}\right)$, and $\mathrm{E}\left(\Omega=2220 \AA^{2}\right)$, in accordance Avith observe the more compact A state for the +9 charge state; however, it is present for lower charge states. At short times the distribution is dominated by the compact $B$ state. This is the only feature observed from 10 to $30 \mathrm{~ms}$, suggesting that ions emerge from the electrospray droplet in relatively compact conformations. Although the cross sections observed are similar to that

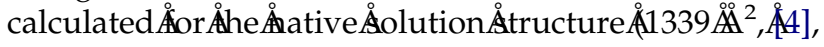
probably this is not the same structure, especially considering that the solution composition from which the ions were electrosprayed would not stabilize the native tructureÅn sive cleavage of the +9 ion, which would not occur if the native solution structure was present in the gas phase: ECD allows intramolecular noncovalent bonds (such as those present in the native folded protein) to remain intact while still dissociating the peptide backbone.

The distribution that elongated conformers ( $\mathrm{D}$ and $\mathrm{E}$ ), as well as a less populated, broad distribution of the $\mathrm{C}$ conformer are formed at longer trapping times. In this case compact ions have unfolded while stored in the trap. The populations of the D and E states decrease by $5000 \mathrm{~ms}$. At the same time, the fraction of ions having $\Omega \sim 1600$ $1800 \AA^{2}$ have increased. Thus, apparently some of the

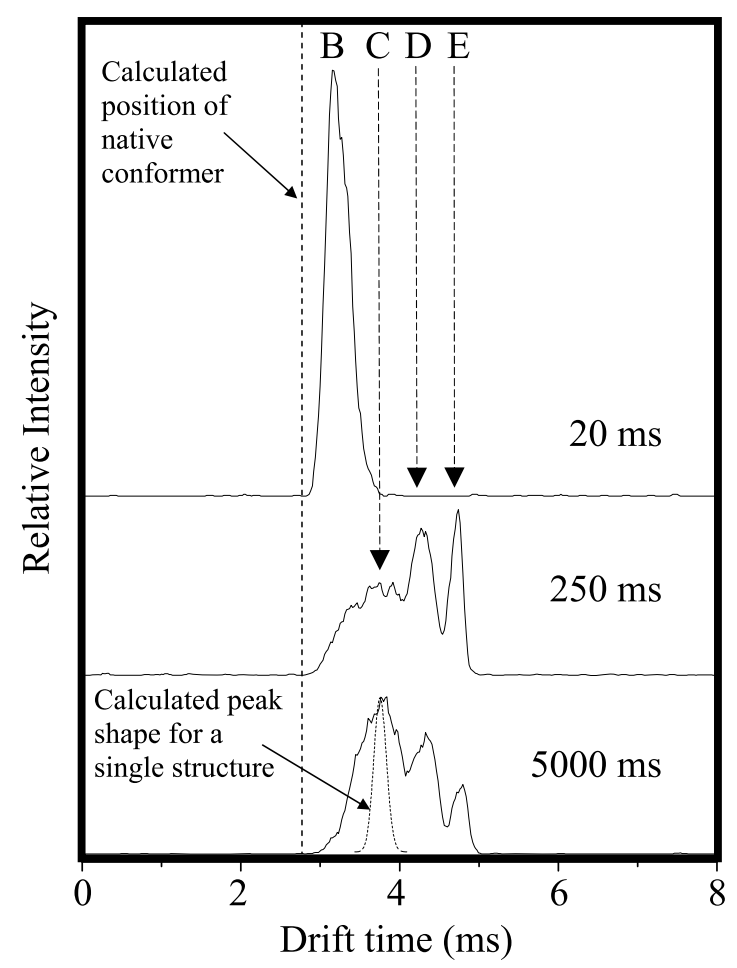

Figure 1. Drift time distributions for the +9 charge state of gas-phase cytochrome $c$ at trapping times of 20, 250, and $5000 \mathrm{~ms}$ showing the ions unfold and refold as a function of time. The different gas-phase conformations are labeled B, C, D, and E, in the order from most compact to most elongated. The dashed line shows the calculated cross section for the native protein using the exact hard-sphere conformers is broader than the dashed peak calculated from the transport equation for a single conformer. The broad peak requires that a distribution of at least four different $\mathrm{F}$ type conformers with similar cross sections is present.

elongated ions ( $\mathrm{D}$ and $\mathrm{E}$ states) have refolded at long storage times. Other charge states observed in these experiments $(+6,+7,+8$, and +10 , not shown) showed unfolding transitions but no evidence of refolding transitions under the conditions studied. They follow sim-

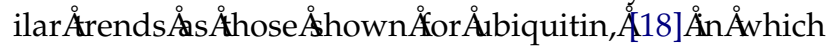
lower charge states show less unfolding over time than higher charge states.

A more detailed understanding of the transitions

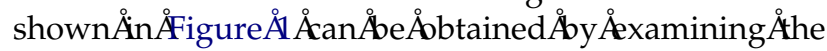
populations of different states over the entire storage time Ånge, Ås Åhown Ån tions of each peak to the total distribution were obtained by integrating the area under each of the conformer peaks observed by using the experimental peak shapes to fit the data. The B, D, and E peaks were fit and subtracted from the total distribution; the remaining relatively broad distribution was assigned to the multiple $\mathrm{C} / \mathrm{F}$ conformers.

The B state rapidly unfolds (by $60 \mathrm{~ms}$ ) to the more elongated structures; however, it does not disappear entirely ( $\sim 10 \%$ of B-type ions persist to very long times), indicating the presence of a second very stable 


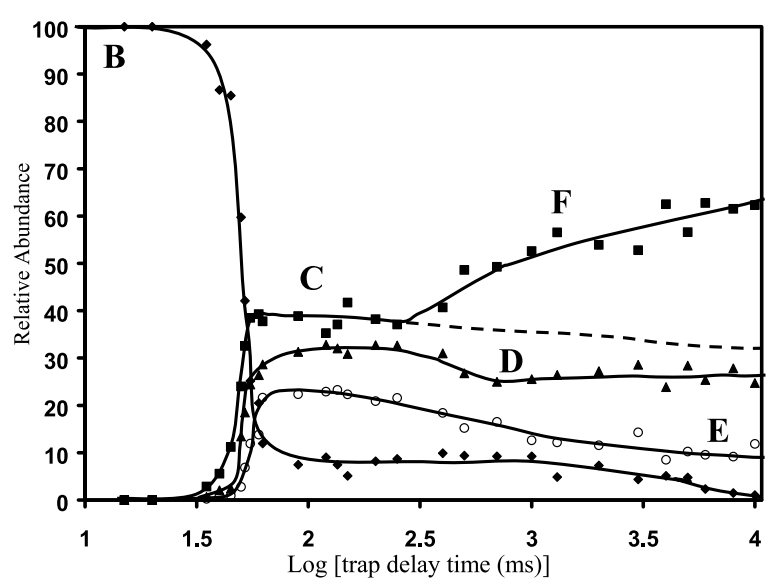

Figure 2. Relative abundance of different conformations of the +9 charge state of cytochrome $c$ as a function of trapping time (plotted on a logarithmic scale). The different states are shown as solid Aiamonds Âstate solid squares (states $\mathrm{C}$ and $\mathrm{F}$ observed at a drift time of $3.77 \mathrm{~ms}$ ), solid triangles (state D at a drift time of $4.33 \mathrm{~ms}$ ), and open circles (state E, having a drift time of $4.74 \mathrm{~ms}$ ). Solid lines through the data are provided only as visual guides. The dashed line is provided to suggest the relative abundance of states $\mathrm{C}$ and $\mathrm{F}$.

conformation having the same cross section as the one that has unfolded. At $250 \mathrm{~ms}$ the $\mathrm{C}$ state is at $37 \%$ abundance and the more elongated D and $\mathrm{E}$ states are at relative abundance of 33 and $21 \%$, respectively.

Between $250 \mathrm{~ms}$ and $5 \mathrm{~s}$, the remaining compact B state has decreased slightly to $5 \%$. The relative abundance of the conformers with cross sections near that of the $\mathrm{C}$ state has increased to $57 \%$, whereas the contributions from the elongated $\mathrm{E}$ state has decreased to $10 \%$ (the D state decreased slightly to $28 \%$ before leveling off). This indicates that the elongated conformers (D and E) have refolded to more compact states. The broad shape of this peak indicates that $\mathrm{D}$ and $\mathrm{E}$ states refold to multiple structures having cross sections that are similar to the $\mathrm{C}$ state. Beginning at $250 \mathrm{~ms}$, the curve for $\mathrm{C}$

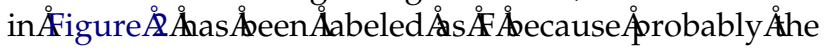
ion population at the drift time of the $C$ state is from a conformer that has a similar cross section but is instead a different structure that can not be resolved from C. We also have performed experiments in which the +9 ion was mass selected in the ion trap as described previously $\AA 23]$, Åduring Åwhich Åhe Åresultant collisional heating drove the ion to unfold and favor primarily the elongated conformers ( $\mathrm{D}$ and $\mathrm{E}$, with a small peak corresponding to $\mathrm{C}$ ). When these ions were stored for extended time periods, they too refolded to the more compact $\mathrm{F}$ states. Thus, there is strong evidence that $\mathrm{F}$ can be formed from D and E, suggesting that this conformation has the same cross section but a different folded structure than $\mathrm{C}$. We can not rule out, however, that $\mathrm{C}$ and $\mathrm{F}$ have similar structures.

The long time associated with formation of the more compact $\mathrm{F}$ state indicates that it does not come directly from the compact B state or directly from solution. Instead, apparently, compact ions must first unfold to relatively extended states before refolding to the more compact F states. Presumably, this is a result of conformational restrictions that prevent the entire population of $\mathrm{B}$ ions from directly forming $\mathrm{F}$. These elongated intermediates may relieve steric restrictions associated with direct conversion from the initial to the subsequent compact states. Additionally, the new structures that are formed appear as a broad peak, indicating that over the timescale that has been studied, refolding in the gas phase is generating new more compact species that have a range of similar cross sections; thus, refolding to this point appears to lead to less specific species than folding of this protein in solution.

We note that we have also performed molecular dynamics calculations for cytochrome $c$ ions (having a number of different charge site assignments) and have calculated many cross sections for the structures that we have observed. In the end, these calculations still have not provided clues that provide more insight about the unfolding and refolding transitions observed here; therefore, we do not show any proposed structures for intermediates or final structures that are observed experimentally. Instead, Scheme 1 uses some hypothetical "cartoon" structures to illustrate the transitions that are observed for the +9 ion on storing electrosprayed ions in a trap. This scheme shows that some states are accessible by multiple pathways $(\mathrm{B} \rightarrow \mathrm{C}$, $\mathrm{D}$, and $\mathrm{E}$ ) while other steps are relatively specific ( $\mathrm{D}$ and $\mathrm{E} \rightarrow \mathrm{F}$ ). This approach can provide information about the timescales and numbers of different states associated with formation of large ions as they emerge from solution and then evolve into new gas-phase structures. Overall, the process in which structure is established in the gas-phase ions shows some similarities with mechanisms for structural formation in denatured to native transitions bserved Ån

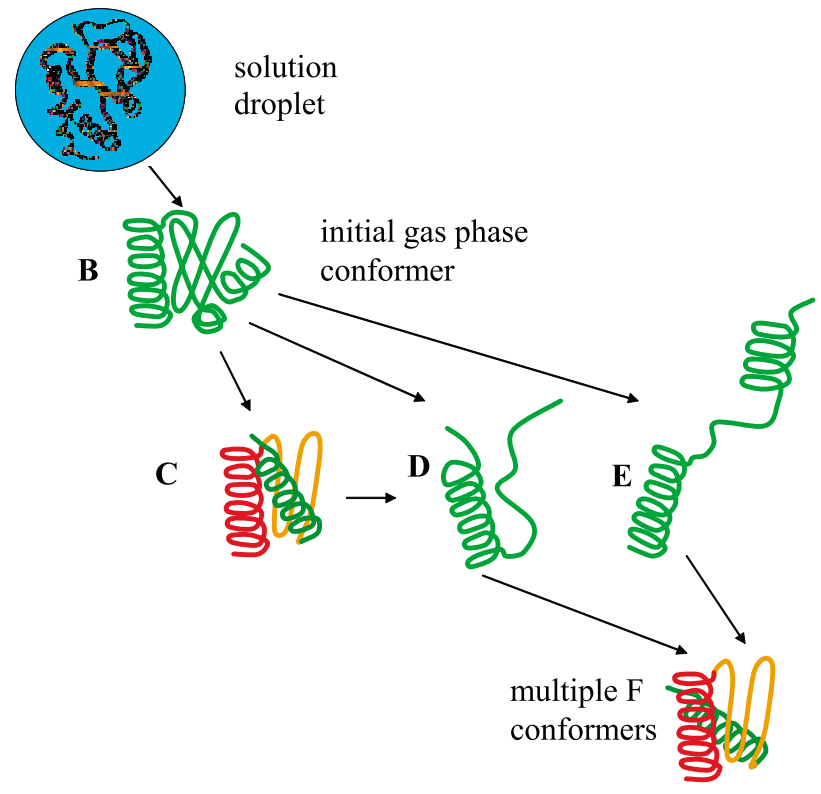

Scheme 1. Proposed pathway for gas-phase refolding of +9 cytochrome $c$. 
An interesting (but still unresolved) issue involves the kinetics associated with the initial unfolding of state B. This process appears to involve an induction period as molecules this is a very unusual behavior and we are aware of no examples of this type of delay before a concerted transition. In an unpublished work (2002), Jarrold and Clemmer have proposed that such a process might involve a large number of small transitions that occur over similar activation barriers, either by sequential or parallel steps; in such a model after a critical number of steps, the compact state becomes unstable and opens up to a more open conformation with a measurably larger cross section. We do not develop this model here because we still have not ruled out the possibility that the internal temperature of the population of ions is influenced during storage in the trap. McLuckey and his coworkers have shown that 10's of milliseconds are required for internal ion temperatures

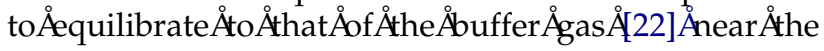
transition times here.

Finally, we note that in this system, timescales for unfolding and refolding are about seconds, rather than microseconds to milliseconds, as observed in solution. The timescales observed in our results are comparable with those obtained earlier by McLafferty and Ácoworkers $A ̊[11]$ Åusing Ålaser-induced Åunfolding studied by ECD. They observed refolding of +9 cytochrome $c$ ions in an Fourier transform ion cyclotron resonance (FT-ICR) cell over a timescale of $120 \mathrm{~s}$, compared with our timescale of $10 \mathrm{~s}$. Their data show that the ECD spectra obtained $120 \mathrm{~s}$ after laser excitation are similar to spectra recorded without laser excitation, confirming that the ions have refolded $120 \mathrm{~s}$ after being denatured. Possibly both of these experiments are observing similar structural changes. Because of the timescale required for the FT-ICR experiments, the B state may be absent from their zero delay spectra, but the C, D, E, and F states may be what are observed during their unfolding and refolding transitions. In addition, recent FT-ICR results show distinct conformations that do not inter-

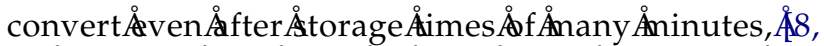

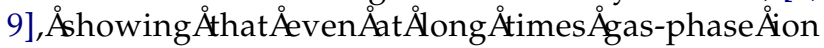
structures have not yet reached equilibrium. We are currently developing variable temperature methods (and also extending these measurements to longer trapping times) to try to find the final favored structures of ions in the gas phase as well as developing a combined labeling and mobility technique to further study the structural transitions observed for cytochrome $c$.

\section{Acknowledgments}

The authors are grateful for many enlightening conversations with Fred McLafferty and Kathrin Breuker and for preprints of their manuscripts. The authors thank Martin F. Jarrold for his input to the model involving the kinetics associated with an initial induction period. This work was supported by a grant from the National Science Foundation (grant CHE-0078737). ERB was supported by a grant to the Indiana University/ Purdue University Indiana Instrumentation Institute (the III) through a grant from the Indiana 21st Century Fund.

\section{References}

1. Bai, Y. W.; Sosnick, T. R.; Mayne, L.; Englander, S. W. Protein Folding Intermediates: Native-State Hydrogen Exchange. Science 1995, 269, 192-197.

2. Dobson, C. M.; Sali, A.; Karplus, M. Protein Folding: A Perspective From Theory and Experiment. Angew. Chem. Int. Ed. Engl. 1998, 37, 868-893.

3. Gruebele, M. The Fast Protein Folding Problem. Annu. Rev. Phys. Chem. 1999, 50, 485-516.

4. Shelimov, K. B.; Clemmer, D. E.; Hudgins, R. R.; Jarrold, M. F. Protein Structure in Vacuo: The Gas Phase Conformations of BPTI and Cytochrome c. J. Am. Chem. Soc. 1997, 119, 22402248.

5. Wyttenbach, T.; Helden, G. V.; Bowers, M. T. Gas-Phase. Conformation of Biological Molecules: Bradykinin. J. Am. Chem. Soc. 1996, 118, 8355-8364.

6. Valentine, S. J.; Clemmer, D. E. H/D Exchange Levels of Shape-Resolved Cytochrome $c$ Conformers in the Gas Phase. J. Am. Chem. Soc. 1997, 119, 3558-3566.

7. Winger, B. E.; Lightwahl, K. J.; Rockwood, A. L.; Smith, R. D. Probing Qualitative Conformation Differences of Multiply Protonated Gas-Phase Proteins via Hydrogen/Deuterium Isotopic Exchange With Water-d2. J. Am. Chem. Soc. 1992, 114, 5897-5898.

8. Wood, T. D.; Chorush, R. A.; Wampler, F. M.; Little, D. P.; Oconnor, P. B.; McLafferty, F. W. Gas-Phase Folding and Unfolding of Cytochrome $c$ Cations. Proc. Natl. Acad. Sci. U.S.A. 1995, 92, 2451-2454.

9. McLafferty, F. W.; Guan, Z. Q.; Haupts, U.; Wood, T. D.; Kelleher, N. L. Gaseous Conformational Structures of Cytochrome c. J. Am. Chem. Soc. 1998, 120, 4732-4740.

10. Freitas, M. A.; Hendrickson, C. L.; Emmett, M. R.; Marshall, A. G. Gas-Phase Bovine Ubiquitin Cation Conformations Resolved by Gas-Phase Hydrogen/Deuterium Exchange Rate and Extent. Int. J. Mass Spectrom. 1999, 187, 565-575.

11. Horn, D. M.; Breuker, K.; Frank, A. J.; McLafferty, F. W. Kinetic Intermediates in the Folding of Gaseous Protein Ions Characterized by Electron Capture Dissociation Mass Spectrometry. J. Am. Chem. Soc. 2001, 123, 9792-9799.

12. Breuker, K.; Oh, H. B.; Horn, D. M.; Cerda, B. A.; McLafferty, F. W. Detailed Unfolding and Folding of Gaseous Ubiquitin Ions Characterized by Electron Capture Dissociation. J. Am. Chem. Soc. 2002, 124, 6407-6420.

13. Zubarev, R. A.; Horn, D. M.; Fridriksson, E. K.; Kelleher, N. L.; Kruger, N. A.; Lewis, M. A.; Carpenter, B. K.; McLafferty, F. W. Electron Capture Dissociation for Structural Characterization of Multiply Charged Protein Cations. Anal. Chem. 2000, $72,563-573$.

14. Woenckhaus, J.; Mao, Y.; Jarrold, M. F. Hydration of Gas Phase Proteins: Folded +5 and Unfolded +7 Charge States of Cytochrome c. J. Phys. Chem. B 1997, 101, 847-851.

15. Jarrold, M. F. Peptides and Proteins in the Vapor Phase. Annu. Rev. Phys. Chem. 2000, 51, 179-207.

16. Woenckhaus, J. Drift Time Mass Spectrometric Protein Hydration Experiments. Int. J. Mass Spectrom. 2002, 213, 9-24.

17. Badman, E. R.; Hoaglund-Hyzer, C. S.; Clemmer, D. E. Monitoring Structural Changes of Proteins in an Ion Trap over 10-200 ms: Unfolding Transitions in Cytochrome $c$ Ions. Anal. Chem. 2001, 73, 6000-6007. 
18. Myung, S.; Badman, E. R.; Lee, Y. J.; Clemmer, D. E. Structural Transitions of Electrosprayed Ubiquitin Ions Stored in an Ion Trap over $\sim 10 \mathrm{~ms}$ to 30 s. J. Phys. Chem. A. 2002, 106, 9976-9982.

19. Hoaglund-Hyzer, C. S.; Li, J. W.; Clemmer, D. E. Mobility Labeling for Parallel CID of Ion Mixtures. Anal. Chem. 2000, 72, 2737-2740.

20. Clemmer, D. E.; Jarrold, M. F. Ion Mobility Measurements and Their Applications to Clusters and Biomolecules. J. Mass Spectrom. 1997, 32, 577-592.

21. Valentine, S. J.; Anderson, J. G.; Ellington, A. D.; Clemmer, D. E. Disulfide-Intact and -Reduced Lysozyme in the Gas
Phase: Conformations and Pathways of Folding and Unfolding. J. Phys. Chem. B 1997, 101, 3891-3900.

22. McLuckey, S. A.; Wells, J. M.; Stephenson, J. L.; Goeringer, D. E. Novel Quadrupole Ion Trap Methods for Characterizing the Chemistry of Gaseous Macro-Ions. Int. J. Mass Spectrom. 2000, 200, 137-161.

23. Badman, E. R.; Myung, S.; Clemmer, D. E. Gas-Phase Separations of Protein and Peptide Ion Fragments Generated by Collision-Induced Dissociation in an Ion Trap. Anal. Chem. 2002, 74, 4889-4894.

24. Shvartsburg, A. A.; Jarrold, M. F. An Exact Hard-Spheres Scattering Model for the Mobilities of Polyatomic Ions. Chem. Phys. Lett. 1996, 261, 86-91. 